\title{
Molecular optomechanics in atomic-scale plasmonic hot spots
}

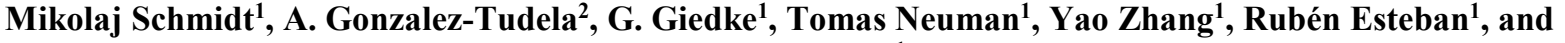 \\ Javier Aizpurua ${ }^{1}$ \\ ${ }^{I}$ Center for Materials Physics (CSIC-UPV/EHU) and Donostia International Physics Center (DIPC), Donostia-San Sebastián, Spain \\ ${ }^{2}$ Max-Planck-Institut fur Quantenoptik Hans-Kopfermann-Str. 1. 85748 Garching, Germany \\ E-mail address: aizpurua@ehu.eus
}

\begin{abstract}
We introduce a Quantum Electrodynamics (QED) approach to describe inelastic scattering processes of molecules in atomic-scale plasmonic picocavities. By solving the corresponding optomechanical dynamics, we identify nonlinear inelastic signals related to vibrational pumping, together with dynamical backaction and strong correlations of the photons emitted.
\end{abstract}

Surface-Enhanced Raman Scattering (SERS) is a fundamental spectroscopic technique that allows to access the rich vibrational structure of molecules. A typical SERS configuration is displayed in Fig. 1(a), with a molecule located in a plasmonic cavity acting as an optical nanoantenna that enhances the vibrational (Stokes or antiStokes) signal. A number of recent implementations of Raman experiments in plasmonic nanocavities appear to provide results which escape the standard description of the Raman process based on the classical treatment of the electromagnetic enhancement of fields inside the cavity [1].

We establish a novel analogy between SERS in molecular spectroscopy and typical optomechanical processes. By adopting an optomechanical hamiltonian, $\hat{H}$, which describes the interaction between cavity plasmons and molecular vibrations, we are able to trace the quantum dynamics of both plasmons and vibrations:

$$
\hat{H}=\omega_{m} \hat{b}^{\dagger} \hat{b}+\omega_{c} \hat{a}^{\dagger} \hat{a}-g \hat{a}^{\dagger} \hat{a}\left(\hat{b}^{\dagger}+\hat{b}\right)+i \Omega\left(\hat{a}^{\dagger} e^{-i \omega_{l} t}-\hat{a} e^{i \omega_{l} t}\right),
$$

where $\omega_{m}$ is the molecular vibration frequency, $\omega_{c}$ is the plasmonic cavity frequency, and $\omega_{l}$ is the incident laser frequency with amplitude $\Omega$. $\hat{a}(b)$ and $\hat{a}^{\dagger}\left(b^{\dagger}\right)$ are the plasmon (molecular vibration) creation and annihilation operators. Importantly, the coupling term $g \hat{a}^{\dagger} \hat{a}\left(\hat{b}^{\dagger}+\hat{b}\right)$ describes the optomechanical interaction, characterized by a coupling parameter $g$ which is proportional to the inverse of the plasmonic effective mode volume [2].

The solution of the master equation of this hamiltonian allows to identify novel quantum effects such as the existence of different regimes of molecular vibrational build-up, as described in Fig. 1(b): a thermal vibrational regime, a vibrational pumping regime, and a strongly nonlinear vibrational regime, which emerge as a consequence of the quantum dynamics induced by the optomechanical interaction. Correlations between the Stokes and anti-Stokes Raman signals can also be traced, as shown in Fig. 1(c).

The presence of strong optomechanical effects in Raman have been recently addressed experimentally in special "picocavities" formed by a few metallic atoms in a plasmonic cavity. The strong optomechanical coupling achieved in this situation is found to activate the pumping regime in the Raman signal [3].

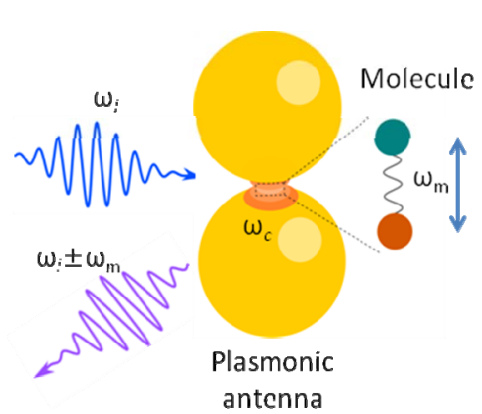

(a)

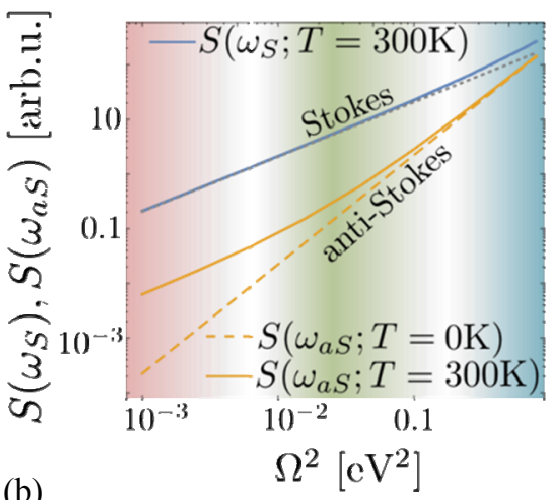

(b)

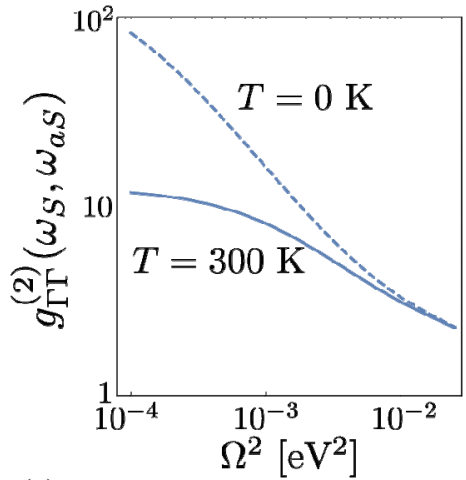

(c)

Fig. 1. (a): Schematics of plasmonic antenna-enhanced molecular optomechanical process for incident laser frequency $\omega_{l}$, molecular vibration frequency $\omega_{m}$, plasmonic cavity frequency $\omega_{c}$, and Stokes or anti-Stokes emission $\left(\omega_{l} \pm \omega_{m}\right)$. (b): Evolution of Stokes $\left(\omega_{\mathrm{S}}\right)$ and antiStokes $\left(\omega_{\mathrm{as}}\right)$ emission as a function of incoming laser power $\Omega^{2}$. Thermal regime (reddish), pumping regime (greenish) and nonlinear regime (bluish). (c): Second-order correlation function of the Stokes and anti-Stokes emission as a function of $\Omega^{2}$ for two temperatures $(0 \mathrm{~K}, 300 \mathrm{~K})$.

\section{References}

[1] R. Zhang, Y. Zhang, Z.C. Dong, S. Jiang, C. Zhang, L.G. Chen, L. Zhang, Y. Liao, J. Aizpurua, Y. Luo, J.L. Yang, and J.G. Hou, Nature 498, 82-86 (2013).

[2] M.K. Schmidt, R. Esteban, A. Gonzalez-Tudela, G. Giedke, J. Aizpurua, ACS Nano 10, 6291-6298 (2016).

[3] F. Benz, M. K. Schmidt, A. Dreismann, R. Chikkaraddy, Y. Zhang, A. Demetriadou, C. Carnegie, H. Ohadi, B. de Nijs, R. Esteban, J. Aizpurua, and J J. Baumberg. Science 354, 726-729 (2016). 